\title{
Isolate-Based Surveillance of Bordetella pertussis, Austria, 2018-2020
}

\author{
Adriana Cabal, Daniela Schmid, Markus Hell, Ali Chakeri, Elisabeth Mustafa-Korninger, \\ Alexandra Wojna, Anna Stöger, Johannes Möst, Eva Leitner, Patrick Hyden, Thomas Rattei, \\ Adele Habington, Ursula Wiedermann, Franz Allerberger, Werner Ruppitsch
}

Pertussis is a vaccine-preventable disease, and its recent resurgence might be attributable to the emergence of strains that differ genetically from the vaccine strain. We describe a novel pertussis isolate-based surveillance system and a core genome multilocus sequence typing scheme to assess Bordetella pertussis genetic variability and investigate the increased incidence of pertussis in Austria. During 2018-2020, we obtained 123 B. pertussis isolates and typed them with the new scheme (2,983 targets and preliminary cluster threshold of $\leq 6$ alleles). $B$. pertussis isolates in Austria differed genetically from the vaccine strain, both in their core genomes and in their vaccine antigen genes; $31.7 \%$ of the isolates were pertactin-deficient. We detected 8 clusters, 1 of them with pertactin-deficient isolates and possibly part of a local outbreak. National expansion of the isolate-based surveillance system is needed to implement pertussis-control strategies.

$B$ ordetella pertussis is the main causative agent of the reemerging respiratory disease commonly known as whooping cough (1). B. pertussis infection usually affects infants, toddlers, and children of school age, although adolescents and adults also can get infected

Author affiliations: Institute for Medical Microbiology and Hygiene, Austrian Agency for Health and Food Safety, Vienna, Austria

(A. Cabal, D. Schmid, A. Chakeri, A. Stöger, F. Allerberger,

W. Ruppitsch); MEDILAB, Teaching Laboratory of the Paracelsus Medical University, Salzburg, Austria (M. Hell, E. MustafaKorninger, A. Wojna); Centre for Public Health, Medical University Vienna, Vienna (A. Chakeri); MB-LAB Clinical Microbiology Laboratory, Innsbruck, Austria (J. Möst); Consultant Laboratory for Bordetella of the Robert Koch Institute, Medical University of Graz, Graz, Austria (E. Leitner); Centre for Microbiology and Environmental Systems Science, University of Vienna, Vienna (P. Hyden, T. Rattei); Children's Health Ireland at Crumlin, Dublin, Ireland (A. Habington); Institute of Specific Prophylaxis and Tropical Medicine, Medical University of Vienna, Vienna (U. Wiedermann)

DOI: https://doi.org/10.3201/eid2703.202314 and have symptoms. In addition, because transmission of pertussis can go unnoticed, asymptomatic carriers are considered an important source of infection (2). Despite its low sensitivity, culturing pertussis from nasopharyngeal swabs remains the standard diagnostic technique, although today it is scarcely performed (3).

To some extent, pertussis can be prevented by vaccination with either cellular or acellular vaccines (4). In Austria, cellular pertussis vaccines were replaced in 1998 by acellular vaccines (ACVs), and ever since, either 2 (filamentous hemagglutinin [FHA] and pertussis toxin [PTX]) or 3 (FHA, PTX, and pertactin) component vaccines have been used for primary vaccination. Booster immunizations have been recommended since 2003 for children of school age and adolescents. These vaccines include either 3 (FHA, PTX, and pertactin) or 5 (FHA, PTX, pertactin, FIM2, and FIM3) components.

Despite vaccinations, the incidence of pertussis has been increasing in the past few decades (5-7). In Austria, 579 pertussis cases were reported in 2015; the number increased to 1,274 in 2016, 1,411 in 2017, 2,198 in 2018, and 2,246 in 2019. The increase in the incidence of pertussis worldwide can be explained partially by the loss of the protective effect after immunity wanes; this loss is strongly associated with ACV use (8). Another factor that contributes to the resurgence of pertussis is the emergence of vaccineevasive $B$. pertussis strains that differ genetically from the vaccine strains $(9,10)$.

A molecular study conducted on pertussis cases from 3 different cities in Austria assessed the genetic variability of $B$. pertussis nationwide (5). However, the study used respiratory samples to perform PCR, followed by Sanger sequencing; therefore, typing was not based on whole-genome sequencing (WGS) of $B$. pertussis isolates. 
Because of the rise in the incidence of pertussis in Austria in recent years, we investigated pertussis cases from 3 states in Austria to assess the genetic variability of their B. pertussis isolates through WGSbased typing. The first objective was to set up a national isolate-based surveillance system, complementary to the case-based surveillance system in Austria, for collecting isolates from patients with suspected pertussis. Second, we aimed to characterize and to compare $B$. pertussis isolates with the vaccine strain Tohama I and other isolates from different geographic regions outside Austria.

\section{Methods}

\section{Setup of the Surveillance System and Sequencing}

For 2 years (May 2018-May 2020), hospitals, general practitioners, and pediatricians using clinical laboratories located in 3 states in Austria (Salzburg, Tyrol, and Styria) were asked to collect $\geq 1$ nasopharyngeal swab containing transport medium (ESwab; Copan, https://www.copangroup.com) from patients with suspected $B$. pertussis infection (Figure 1). When possible, a second nasopharyngeal swab containing charcoal-based medium (Transystem Amies medium with charcoal; Copan) was collected.The swabs were then sent to the clinical laboratory of each state participating in the study. For each suspected case, PCR was performed using the swab containing the transport medium with a commercial kit (BD MAX, Becton Dickinson, http://bd.com; or BORDETELLA R-gene, bioMérieux, https://www.biomerieux. com). When PCR results were positive, either the same swab used for PCR or, if available, the charcoal swab was stroked on Oxoid Bordetella-selective medium (Thermo Fisher Scientific, https://www. thermofisher.com) or Bordet Gengou agar with 15\% sheep blood (Becton Dickinson), followed by cultivation at $37^{\circ} \mathrm{C}$ under aerobic and humid conditions for 48-120 hours. Colonies compatible with B. pertussis were tested by MALDI Biotyper software version 3.0 (Bruker, https://www.bruker.com) or VitekMS software version 3.2 (bioMérieux). Colonies indentified as B. pertussis were sent to the Austrian Agency for Health and Food Safety in Vienna for further DNA extraction and 300-bp paired-end WGS using an Illumina Miseq device (https://www.illumina. com), as described in Appendix 1 (https://wwwnc. cdc.gov/EID/article/27/3/20-2314-App1.pdf). Additional information on the sequencing process, de novo assembly, and sequence quality checks also are found in Appendix 1. The Illumina reads of the 123 isolates in Austria have been deposited in the National Center for Biotechnology Information (NCBI) Sequence Read Archive repository under project number PRJNA642701.

\section{Generation of a B. pertussis cgMLST Scheme}

A stable, ad hoc, core-genome multilocus sequence typing (cgMLST) scheme and accessory genome scheme were created by using Ridom SeqSphere + version 4.1 .9 (Ridom, https://www.ridom.de; Appendix 1). In brief, 15 genomes (Appendix 2 Table 1, https://wwwnc.cdc.gov/EID/article/27/3/202314-App2.xlsx) were used as query genomes and the Tohama I vaccine strain genome (GenBank accession no. NC_002929.2) as a seed genome. Afterwards, 263 taxonomic and quality outliers were discarded, leaving a total of 2,983 core genome targets (Appendix 2 Table 2) and 179 accessory genome targets (Appendix 2 Table 3). We considered as core genes only those targets (i.e., genes) that were present in $100 \%$ of the genomes. Further validation of the scheme was based on a selection of B. pertussis genomes available in NCBI ( $\mathrm{n}=391)$, many of which were associated with outbreaks (Appendix 2 Table 4 ), and an old collection of clinical Bordetella sp. strains from Austria (Appendix 2 Table 5).

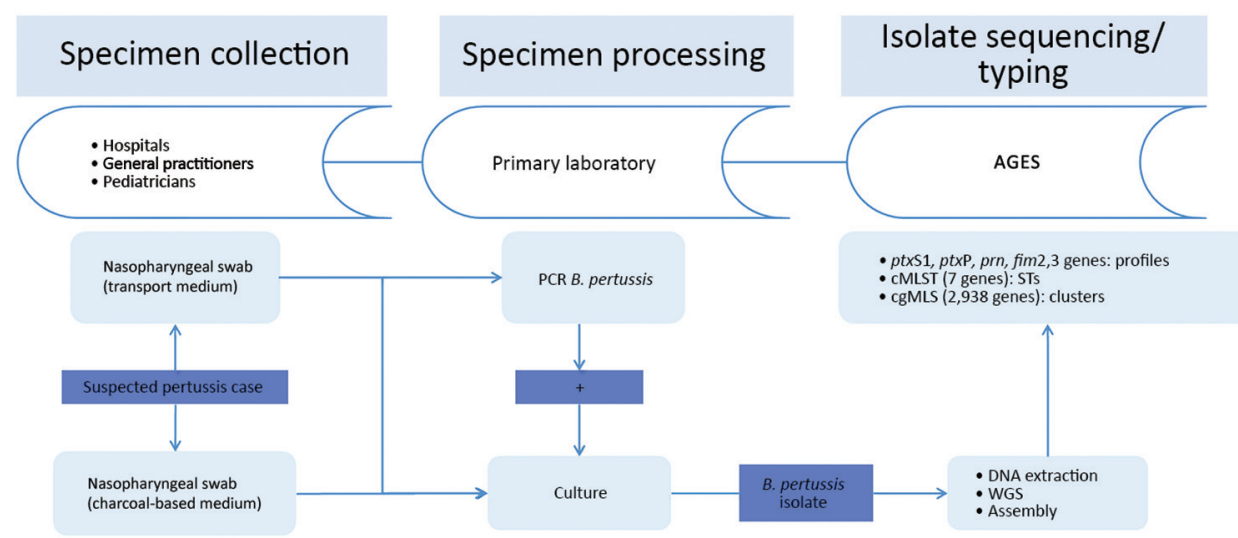

Figure 1. Flow chart of the Bordetella pertussis isolatebased surveillance system, Austria, May 2018-May 2020. AGES, Agentur für Gesundheit und Ernährungssicherheit (Austrian Agency for Health and Food Safety); cgMLST, coregenome multilocus sequence typing; ST, sequence type. 
Typing of B. pertussis Isolates and Comparative Analysis During the 2-year study period, all the clinical isolates collected within the isolate-based surveillance system were typed with our newly implemented cgMLST scheme. Allelic differences among the isolates from Austria and the vaccine strain Tohama I were visualized by generating minimum spanning trees with a preliminary cluster threshold established at $\leq 6$ alleles (Appendix 1). We extracted the sequence types (STs) from the WGS data corresponding to the classical multilocus sequence typing (11), the variants and mutations present in each of the genes used as vaccine antigens ( $p t x \mathrm{~S} 1, p t x \mathrm{P}, p r n$, fim 2, fim 3$)$, and their combination (genetic profiles).

To be certain that our scheme could be applied beyond our set of B. pertussis isolates from Austria, we used a selection $(n=106)$ of $B$. pertussis genomes, including outbreak strains used in the validation of the cgMLST scheme, to perform a genomic comparative analysis (Appendix 1; Appendix 2 Table 4). We compared the gene content obtained for our cgMLST

\begin{tabular}{|c|c|}
\hline Characteristic & No. \\
\hline \multicolumn{2}{|l|}{ Age group, $y$} \\
\hline$<1$ & 8 \\
\hline $1-4$ & 15 \\
\hline $5-9$ & 31 \\
\hline $10-14$ & 31 \\
\hline $15-19$ & 7 \\
\hline $20-29$ & 3 \\
\hline 30-39 & 7 \\
\hline $40-49$ & 11 \\
\hline $50-59$ & 3 \\
\hline$\geq 60$ & 7 \\
\hline \multicolumn{2}{|l|}{ Sex } \\
\hline $\mathrm{F}$ & 69 \\
\hline M & 54 \\
\hline \multicolumn{2}{|l|}{ State } \\
\hline Salzburg & 86 \\
\hline Tyrol & 21 \\
\hline Styria & 12 \\
\hline Upper Austria & 4 \\
\hline \multicolumn{2}{|l|}{ Clinical symptoms } \\
\hline Coughing fits & 66 \\
\hline Cough >4 weeks & 64 \\
\hline Medical whooping cough diagnosis & 37 \\
\hline Missing data & 7 \\
\hline Post-coughing vomiting & 7 \\
\hline Inspiratory whooping & 5 \\
\hline Asymptomatic & 2 \\
\hline \multicolumn{2}{|l|}{ Vaccination status } \\
\hline Vaccinated & 53 \\
\hline 1st booster & 1 \\
\hline 2nd booster & 22 \\
\hline 3rd booster & 16 \\
\hline 4th booster & 7 \\
\hline Unknown doses & 7 \\
\hline Not vaccinated & 39 \\
\hline Unknown & 31 \\
\hline
\end{tabular}

scheme with the cgMLST scheme developed by the Pasteur Institute (Paris, France) (12). In addition, we compared the results obtained when applying our cgMLST with those derived from a single-nucleotide polymorphism (SNP)-based analysis on the 123 isolates from Austria (Appendix 1).

\section{Statistical Analysis}

Personal information and vaccination status were obtained for each pertussis culture-positive case-patient from the national electronic reporting system. We calculated odds ratios with Stata software version 13 (StataCorp, https://www.stata.com) to measure for associations between pertactin deficiency and vaccination status. In the analysis, we included all case-patients who had received $\geq 1$ dose of pertussis vaccine and those reported as unvaccinated. Case-patients with an unknown vaccination status $(n=31)$ were excluded from the analysis. Statistical significance was defined as $\mathrm{p}<0.05$ by using the Pearson $\chi^{2}$ test or Fisher exact test.

\section{Results}

\section{Culture-Positive Cases}

At the Austrian Agency for Health and Food Safety, we received $123 \mathrm{~B}$. pertussis isolates, collected from 123 pertussis case-patients (Table 1), through our newly implemented isolate-based pertussis surveillance system during May 2018-May 2020. Fewer than $20 \%$ of the total pertussis cases reported in Salzburg state $(\mathrm{n}=310)$ in 2018 were estimated to be culturepositive, and no information on the proportion of cases with a positive pertussis culture was available for the other 8 states in Austria.

A total of $119 \mathrm{~B}$. pertussis isolates belonged to patients with PCR-positive confirmed pertussis from Salzburg, Tyrol, and Styria (Figure 2), and 4 isolates belonged to pertussis case-patients identified in the state of Upper Austria, provided by a clinical microbiology laboratory located in Salzburg (MB-LAB Clinical Microbiology Laboratory). Overall, 15 B. pertussis isolates belonged to culture-positive pertussis case-patients who lived in the same household with $\geq 1$ other culture-positive case-patient. Additional metadata for the 123 pertussis cases are presented in Appendix 2 Table 6.

\section{Sequence Types and Typing of Vaccine Target Genes}

The $B$. pertussis isolates obtained from the 123 pertussis patients in Austria differed in sequence type and in the vaccine antigen genes from the vaccine strain Tohama I (Table 2). We detected ST2 for all but 1 


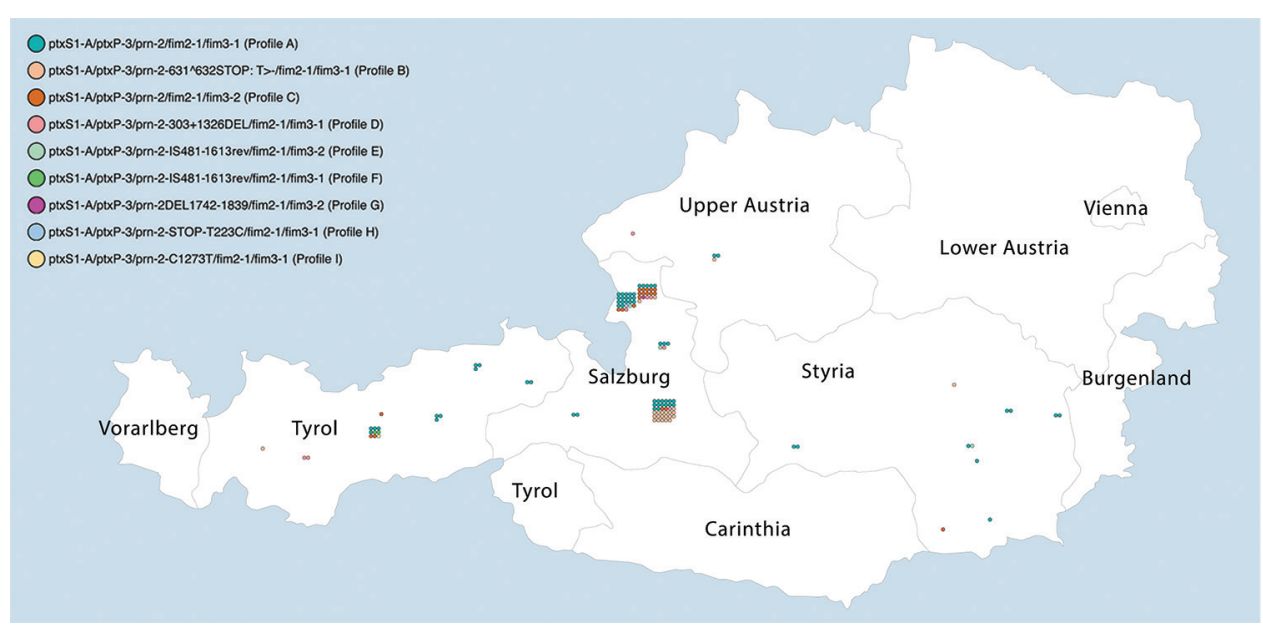

Figure 2. Pertussis cases by district of residence of casepatient and genetic profile of the corresponding Bordetella pertussis isolate identified in a $B$. pertussis isolate-based surveillance study, Austria, May 2018-May 2020. Each dot represents 1 case. Cases grouping next to each other belong to the same district. To protect patient confidentiality, only states and not districts are labeled.

isolate, which was of ST83. We found 9 different genetic profiles (A-I), 1 of which was new (profile G) (Table 2; Figure 3; Appendix 2 Table 7).

We found 7 pertactin-deficient profiles (B and D-I), representing $31.7 \%(n=39)$ of the isolates, and 6 different known pertactin inactivation mechanisms (13-16; Table 2; Appendix 2 Table 7). Pertactin-deficient isolates were mostly of profile $B(n=23$ [18.7\%]). Twenty case-patients (51.3\%) with pertactin-deficient isolates had been vaccinated, $11(28.2 \%)$ case-patients were unvaccinated, and for $8(20.5 \%)$ case-patients, vaccination status was unknown. Case-patients having received $\geq 1$ dose of pertussis $\mathrm{ACV}$ were 1.5 times more likely to have a pertactin-deficient $B$. pertussis isolate (of any genetic profile) compared with unvaccinated case-patients, although this relationship was not statistically significant (unadjusted odds ratio 1.5, 95\% CI 0.6-3.8). Persons living in the district of St. Johann in Pongau (Salzburg state) were 21.17 (95\% CI 6.7-81.1) times more likely to have profile B, and this association was significant $(p<0.001)$. Stratifying by vaccination status, vaccinated persons from St. Johann in Pongau were 13.3 (95\% C: 2.9-99.1; p<0.001) times more likely to present profile B, whereas unvaccinated ones had 58.5 (95\% CI 5.6-1876; $p<0.001)$ times more chances to present profile B. No association was seen between the different age groups or having a pertactin-deficient profile and having profile $B$.

\section{cgMLST and Comparative Analysis}

The 123 B. pertussis isolates were closely related, differing by a maximum of 38 alleles ( $\leq 44$ alleles when including the accessory genome), and they seemed to cluster in groups (Figure 3). We observed that we could separate fim 3-1 and fim3-1 isolates into 2 branches (Figure 4) and that pertactin-deficient isolates of genetic profile $B$ grouped together. Isolates of profile $B(n=23)$ differed by a maximum of 9 alleles when including the only isolate from Tyrol of that profile, and by $\leq 6$ alleles, excluding the isolate from Tyrol. All other isolates were from Salzburg $(n=20)$, Styria $(n=1)$, and Upper Austria $(n=1)$. Isolates from profile $\mathrm{D}$ also clustered together ( $\leq 6$ alleles), differing by $\geq 8$ alleles with isolates of profiles A and B.

With the preliminary cluster threshold of $\leq 6$ alleles, we distinguished 8 clusters (Figure 3; Appendix

Table 2. Genetic profiles of the 123 Bordetella pertussis isolates obtained through the $B$. pertussis isolate-based surveillance system, Austria, May 2018-May 2020*

\begin{tabular}{|c|c|c|c|c|c|c|c|}
\hline \multirow[b]{2}{*}{ Profile } & \multirow[b]{2}{*}{ No. (\%) } & \multicolumn{5}{|c|}{ B. pertussis vaccine antigen genes } & \multirow[b]{2}{*}{ Reference } \\
\hline & & ptxS1 & $p t x \mathrm{P}$ & prn & fim2 & fim3 & \\
\hline Profile vaccine strain Tohama I & 0 & $p t x S 1-D$ & ptxP-1 & prn-1 & fim2-1 & fim3-1 & NA \\
\hline Profile A & $64(52.3)$ & ptxS1-A & $p t x \mathrm{P}-3$ & prn-2 & fim2-1 & fim3-1 & $(13,14)$ \\
\hline Profile B & $23(18.7)$ & ptxS1-A & $p t x \mathrm{P}-3$ & prn-2-631^632STOP:T>- & fim2-1 & fim3-1 & (15) \\
\hline Profile C & $20(16.2)$ & ptxS1-A & $p t x \mathrm{P}-3$ & prn-2 & fim2-1 & fim3-2 & $(13,14)$ \\
\hline Profile D & $8(6.50)$ & ptxS1-A & $p t x \mathrm{P}-3$ & prn-2-303+1326DEL & fim2-1 & fim3-1 & $(16)$ \\
\hline Profile E & $3 \dagger(2.44)$ & $p t x S 1-A$ & $p t x \mathrm{P}-3$ & prn-2-IS481-1613rev & fim2-1 & fim3-2 & $(13,14)$ \\
\hline Profile F & $2(1.62)$ & $p t x S 1-A$ & $p t x \mathrm{P}-3$ & prn-2-IS481-1613rev & fim2-1 & fim3-1 & $(14)$ \\
\hline Profile G & $1(0.8)$ & $p t x S 1-A$ & $p t x \mathrm{P}-3$ & prn-2- DEL1742-1839ł & fim2-1 & fim3-2 & This study \\
\hline Profile $\mathrm{H}$ & $1(0.8)$ & $p t x S 1-A$ & ptxP-3 & prn-2-STOP-T223C & fim2-1 & fim3-1 & (14) \\
\hline Profile I & $1(0.8)$ & ptxS1-A & $p t \times \mathrm{P}-3$ & prn-2-C1273T & fim2-1 & fim3-1 & $(13,14)$ \\
\hline
\end{tabular}



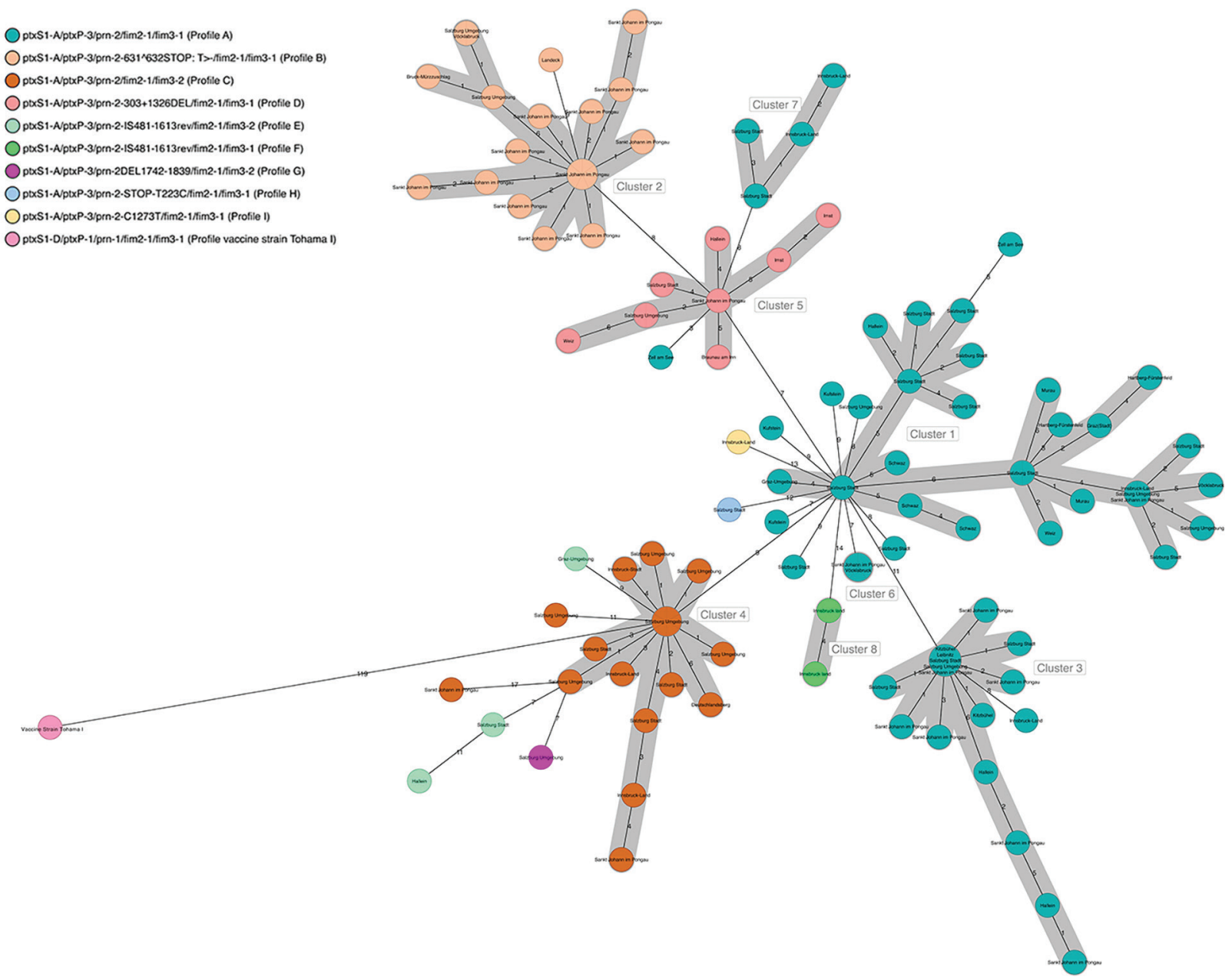

Figure 3. Minimum spanning tree of 123 Bordetella pertussis isolates and their clusters by genetic profile based on core-genome multilocus sequence typing in a B. pertussis isolate-based surveillance study, Austria, May 2018-May 2020. Numbers on connection lines represent the number of allelic differences among the isolates.

1). Cluster 1 integrated only profile A isolates $(n=26)$ from all states. Isolates in cluster $2(n=22)$ were of profile B. Most of the isolates in this cluster $(n=20)$ originated from case-patients living in Salzburg state. Eighteen of them resided in the district of St. Johann in Pongau. Of the 2 case-patients who did not live in Salzburg state, 1 had a confirmed epidemiologic link with a pertussis-positive relative in Salzburg. A trend compatible with a local outbreak of genetic profile B B. pertussis was distinguished (Appendix 1 Figure 1) for this cluster when we compared the number of profile B cases with the total number of reported cases (culture positive and nonculture positive) in the same period for St. Johann in Pongau $(\mathrm{n}=160)$. In addition, a peak of cases in November 2018 corresponded to a small peak of cases with genetic profile B isolates. Cluster 3 had 19 isolates of profile A that were ob- tained from case-patients living in Salzburg, Tyrol, and Styria. Cluster 4 had 18 isolates of profile $C$ from Salzburg, Tyrol, and Styria. Cluster 5 consisted of 9 profile D isolates from Salzburg, Styria, Tyrol, and Upper Austria. Cluster 6 included 5 profile A isolates from Salzburg and Upper Austria, and cluster 7 combined 4 profile A isolates from Salzburg and Tyrol. Cluster 8 had only 2 isolates, both of profile F, which originated from case-patients from Tyrol. Isolates from profiles E, G, H, and I did not cluster with any other isolate. The ST83 isolate did not cluster with any other isolate and differed from isolates in cluster 4 by $\geq 9$ alleles.

In 6 households (A-E and G) (Appendix 2 Table 8 ), we confirmed the transmission of the same B. pertussis strain between 2 or 3 household members with a maximum of 4 allelic differences and an identical 
genetic profile. In 1 household $(\mathrm{F})$, transmission of $B$. pertussis was ruled out when cgMLST revealed 18 allelic differences between 2 isolates obtained from 2 case-patients living together, each of them with a $B$. pertussis strain of a different genetic profile (A and I).

We developed a comparative analysis between our 123 B. pertussis isolates and 106 B. pertussis genomes from NCBI, including mostly isolates from the United States and United Kingdom (Appendix 2 Table 4) from the 2010 and 2012 epidemics $(13,14,16)$ (Figure 4). All fim3-1 isolates were clearly separated from fim3-2 isolates, but we could also differentiate isolates from the same country in different branches. On the basis of the prn type, which comprised the prn wild type alleles or mutations in this gene (i.e., insertions, deletions, and truncations), we distinguished isolates grouping closely to each other, sometimes originating from different countries. Genetic profiles A, C, H, and I were also represented in strains from outside Austria.

We compared target content between the Pasteur Institute's cgMLST scheme and ours (Appendix 2 Table 9). A total of 1,749 genes were common to both schemes; 1,239 genes were only present in our scheme, and 294 genes were only present in Pasteur Institute's scheme. The SNP analysis revealed isolates grouping in clusters in a similar way to that resulting from the cgMLST analysis (Appendix 1 Figure 2).

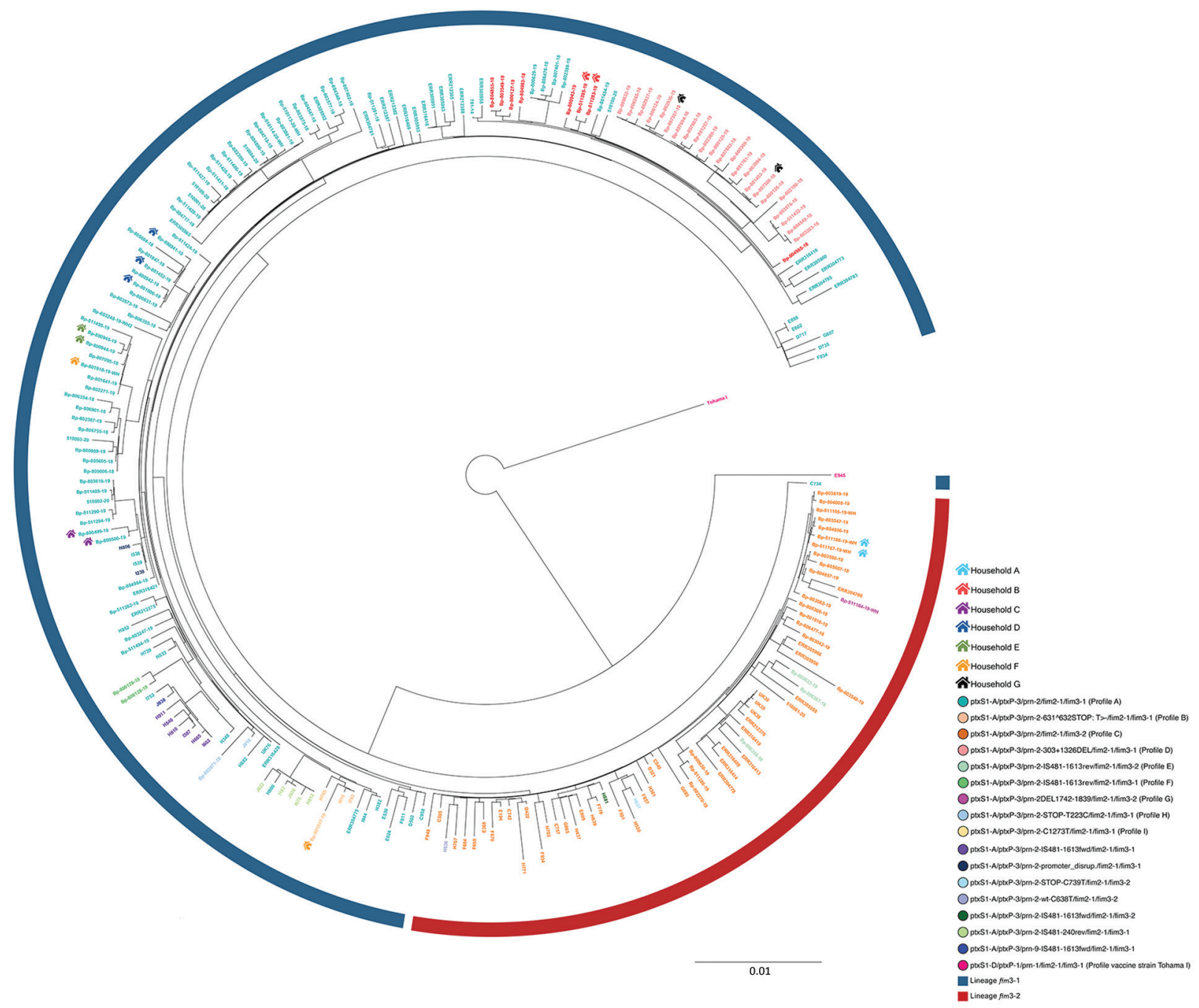

Figure 4. Maximum-likelihood phylogenetic tree generated using core-genome multilocus sequence typing data from 106 outbreak genome sequences from the United States and the United Kingdom and the 123 Bordetella pertussis isolates identified in an isolatebased surveillance study, Austria, May 2018-May 2020. Isolate identifiers are colored by genetic profile. These genetic profiles include profiles A-I, defined in this study, and other genetic profiles described outside of Austria. The circular blue line represents isolates of fim3-1 lineage; the circular red line represents fim3-1 isolates. A color-coded house-like symbol indicates isolates obtained from casepatients living in the same household. Scale bar indicates nucleotide substitutions per site. 


\section{Discussion}

Our newly implemented B. pertussis isolate-based surveillance system has contributed to a better understanding of the molecular epidemiology of pertussis in Austria. No relationship existed between the pertussis incidence in the 3 states in Austria and the number of isolates collected, which depended mainly on the expertise of the respective laboratories in obtaining B. pertussis cultures. The estimated proportion $(\approx 20 \%)$ of culture-positive pertussis cases found in relation to the total number of pertussis cases reported in Salzburg state was consistent with previous reports (17), whereas other authors reported up to $30 \%$ (18).

Results retrieved from the typing of the $123 \mathrm{~B}$. pertussis isolates revealed the presence of the $p t x P-3$ allele in all isolates tested, consistent with other studies (19-21), thereby clearly indicating a divergence from the vaccine strain in Austria and a substitution among currently circulating $B$. pertussis strains of the $p$ txP-1 allele by the $p t x P-3$ allele. In comparison, during 2002-2008, a previous study in Austria still detected the $p t x P-1$ allele in $7 \%$ of the samples (5). Previous data also showed most of $B$. pertussis isolates $(\approx 80 \%)$ grouping in the fim $3-1$ clade, which is more ancestral than fim3-2 (22). Therefore, not surprisingly, the genetic profile A (fim3-1 clade) was one of the most frequently detected genetic profiles globally (22-24), consistent with the findings of our study.

As for the proportion of pertactin-deficient isolates detected $(31.7 \%)$, this finding was similar to the frequency reported during 2012-2015 in Norway, where ACVs were also introduced in 1998 and booster doses recommended after 2001 (15). In contrast, up to $98 \%$ of pertactin-deficient isolates were reported outside the European Union (25). In general, the proportion of $B$. pertussis isolates with pertactin deficiency seemed to be vary among countries depending on the vaccination schedule and vaccine type used (26). Those countries still using cellular pertussis vaccines have never or rarely reported pertactin-deficient isolates $(27,28)$, whereas countries using ACVs have seen a direct association between the year of introduction of ACVs in the country and the appearance of pertactin-deficient isolates (15). Moreover, ACV-vaccinated persons seem more susceptible to pertactin-deficient strains than to pertactin-producing strains, given that pertactin-deficient strains are better able to colonize the respiratory tract (29). According to some authors, immunization with 2-component ACVs (instead of an ACV with 4 or 5 components) might affect immunogenicity (30-32). However, more time is needed to evaluate whether the lack of the pertactin component of 1 of the ACVs affects the incidence of pertussis in Austria in the coming years. Nevertheless, the higher likelihood of profile B strains found in the St. Johann in Pongau district seems not to have been influenced by vaccination.

Regarding the mechanisms causing pertactin deficiency, we reported a mutation at the $632 \mathrm{nt}$ of the prn gene for our profile B isolates, previously described for isolates collected in Italy, Sweden, and Denmark during 2012-2015 (15). Likewise, this deletion was reported in Ireland in 2016 in an isolate (GenBank accession no. KX462969.1) differing from the isolates of cluster 2 in Austria by only 8 alleles. On the contrary, the mutation $\mathrm{T} 223 \mathrm{C}$ in profile $\mathrm{H}$ had been previously reported during 2012-2015 in Australia, the Netherlands, Norway, Sweden, the United Kingdom, and the United States (33-35). The mutation in the prn gene at nt 1326 was reported in the United Kingdom (16) in 2012. Similar mutations at nt 1325 and 1340 of the prn gene were also detected in isolates from Australia (35) and the United States (36). Likewise, the mutation at nt 1273 had been previously found in Canada (37) and the United States as well $(13,14)$. Last, the insertion of the IS481 at nt 1613 in reverse direction was also reported in Canada and the United States $(13,14)$.

The preliminary cluster threshold proposed in this study has served to delineate clusters and can be adjusted when more epidemiologic data derived from contact tracing are available. Establishing a fixed cluster threshold for $B$. pertussis is challenging also because of its homogeneous core genome. Also, because the bacterium undergoes large genomic rearrangements that are only detectable with advanced bioinformatics (14), this diversity might not be captured by cgMLST alone. A possibility to increase the typing resolution obtained with cgMLST for detecting pertussis outbreaks might be to investigate the distribution of IS481 within the B. pertussis genome, as proposed elsewhere (14). In either case, cgMLST allowed the identification of a cluster (cluster 2) of pertactin-deficient isolates from casepatients living in St. Johann in Pongau, possibly indicating the presence of a local pertussis outbreak. We could not determine whether all cases occurring within the period of detection of the genetic profile $B$ belonged to that profile or to another genetic profile because we did not receive $B$. pertussis cultures for each pertussis case. Except for cluster 2, we could neither confirm nor refute that all clusters identified in our study represented single outbreaks. However, we demonstrated the direct transmission of the same pertussis strain by cgMLST among members of the same household. We hypothesize that the 2 casepatients in the household where 2 different genetic 
profiles and cgMLST (18 alleles of difference) were detected might have acquired pertussis from different sources.

Our results of the comparative genomic analysis using global strains were concordant with other studies, in which diverse B. pertussis genetic profiles are shown to be distributed across countries $(12-14,16)$. The pertactin-deficient strains seemed not to belong to the same clone and the mutations observed in each country might consist of independent mutations, as previously described (38). In the absence of more sequences to compare with our isolates in Austria, profile B isolates seemed to be found only in Austria, although the mutation prn-2631^632STOP:T>- had already been reported (15). In addition, the number of allelic differences between isolates not geographically nor temporally related to the isolates in Austria was sometimes as low as 2, matching recently reported findings (12).

The small differences observed between the SNPbased analysis and the cgMLST-based analysis might be attributable to the slightly higher discriminatory power of SNP-based analysis (39). Conversely, the differences in gene content between the Pasteur Institute's cgMLST scheme and ours indicate that our cgMLST was more discriminatory and therefore more suitable for cluster detection in Austria. The uneven number of loci composing each cgMLST might be partially attributable to the different algorithms used by Seqsphere and BIGSdb (40) but also to the fact that only targets that were present in all query genomes were included as targets of our cgMLST scheme. In contrast, the Pasteur Institute's scheme included targets present in $\geq 95 \%$ of all query genomes. Bouchez et al. (12) did not define a threshold for their cgMLST scheme, and hence both schemes might not be comparable in terms of isolate clustering.

The main limitation of our study was the incomplete information on the vaccination status of the case-patients and other epidemiologic data, which prevented better assessment of the effects of these genetic shifts on pertussis incidence. Because of the reduced sample size, whether detecting pertactindeficient strains is linked to an increase in pertussis incidence is unclear; therefore, expanding the isolate-based surveillance system at the national level is advisable.

In summary, we found that B. pertussis strains in Austria differ genetically from the vaccine strain, both in their core genomes and their vaccine antigen genes. Furthermore, our cgMLST method has proven to be stable enough to be applied beyond our set of $123 \mathrm{~B}$. pertussis isolates and proven useful to confirm transmission chains among household members and to detect 8 clusters, 1 of which indicated a possible local outbreak. To detect pertussis outbreaks and target pertussis-control strategies, we recommend performing genomic surveillance of $B$. pertussis using the proposed cgMLST scheme with a preliminary cluster threshold of $\leq 6$ alleles, typing data on the vaccine antigen genes, and completing epidemiologic information on pertussis cases.

\section{About the Author}

Dr. Cabal Rosel is a postdoctoral researcher working as a public health microbiologist at the Austrian Agency for Health and Food Safety in Vienna, Austria. Her research interests include microbial genomics, One Health, antimicrobial resistance, and epidemiology.

\section{References}

1. Kilgore PE, Salim AM, Zervos MJ, Schmitt HJ. Pertussis: microbiology, disease, treatment, and prevention. Clin Microbiol Rev. 2016;29:449-86. https://doi.org/10.1128/ CMR.00083-15

2. Paisley RD, Blaylock J, Hartzell JD. Whooping cough in adults: an update on a reemerging infection. Am J Med. 2012;125:141-3. https:// doi.org/10.1016/j.amjmed. 2011.05.008

3. Lee AD, Cassiday PK, Pawloski LC, Tatti KM, Martin MD, Briere EC, et al.; Clinical Validation Study Group. Clinical evaluation and validation of laboratory methods for the diagnosis of Bordetella pertussis infection: culture, polymerase chain reaction $(\mathrm{PCR})$ and anti-pertussis toxin $\mathrm{IgG}$ serology (IgG-PT). PLoS One. 2018;13:e0195979. https://doi.org/10.1371/journal.pone.0195979

4. Mir-Cros A, Moreno-Mingorance A, Martín-Gómez MT, Codina G, Cornejo-Sánchez T, Rajadell M, et al. Population dynamics and antigenic drift of Bordetella pertussis following whole cell vaccine replacement, Barcelona, Spain, 1986-2015. Emerg Microbes Infect. 2019;8:1711-20. https:/ / doi.org/ 10.1080/22221751.2019.1694395

5. Wagner B, Melzer H, Freymüller G, Stumvoll S, Rendi-Wagner P, Paulke-Korinek M, et al. Genetic variation of Bordetella pertussis in Austria. PLoS One. 2015;10:e013262.

6. Latasa P, García-Comas L, Gil de Miguel A, Barranco MD, Rodero I, Sanz JC, et al. Effectiveness of acellular pertussis vaccine and evolution of pertussis incidence in the community of Madrid from 1998 to 2015. Vaccine. 2018;36:1643-9. https:// doi.org/10.1016/j.vaccine.2018.01.070

7. Esposito S, Stefanelli P, Fry NK, Fedele G, He Q, Paterson P, et al.; World Association of Infectious Diseases and Immunological Disorders (WAidid) and the Vaccine Study Group of the European Society of Clinical Microbiology and Infectious Diseases (EVASG). Pertussis prevention: reasons for resurgence, and differences in the current acellular pertussis vaccines. Front Immunol. 2019;10:1344. https://doi.org/10.3389/fimmu.2019.01344

8. Burdin N, Handy LK, Plotkin SA. What is wrong with pertussis vaccine immunity? The problem of waning effectiveness of pertussis vaccines. Cold Spring Harb Perspect Biol. 2017;9:a029454. https://doi.org/10.1101/ cshperspect.a029454 
9. Chiappini E, Stival A, Galli L, de Martino M. Pertussis re-emergence in the post-vaccination era. BMC Infect Dis. 2013;13:151. https://doi.org/10.1186/1471-2334-13-151

10. Mooi FR, van Oirschot H, Heuvelman K, van der Heide HGJ, Gaastra W, Willems RJL. Polymorphism in the Bordetella pertussis virulence factors P.69/pertactin and pertussis toxin in the Netherlands: temporal trends and evidence for vaccine-driven evolution. Infect Immun. 1998;66:670-5. https:/ / doi.org/10.1128/IAI.66.2.670-675.1998

11. Diavatopoulos DA, Cummings CA, Schouls LM, Brinig MM, Relman DA, Mooi FR. Bordetella pertussis, the causative agent of whooping cough, evolved from a distinct, humanassociated lineage of B. bronchiseptica. PLoS Pathog. 2005;1:e45. https://doi.org/10.1371/journal.ppat.0010045

12. Bouchez V, Guglielmini J, Dazas M, Landier A, Toubiana J, Guillot S, et al. Genomic sequencing of Bordetella pertussis for epidemiology and global surveillance of whooping cough. Emerg Infect Dis. 2018;24:988-94. https:/ / doi.org/10.3201/ eid2406.171464

13. Bowden KE, Weigand MR, Peng Y, Cassiday PK, Sammons S, Knipe K, et al. Genome structural diversity among 31 Bordetella pertussis isolates from two recent U.S. whooping cough statewide epidemics. MSphere. 2016; 1:e00036-16. https:// doi.org/10.1128/mSphere.00036-16

14. Weigand MR, Peng Y, Loparev V, Batra D, Bowden KE, Burroughs $\mathrm{M}$, et al. The history of Bordetella pertussis genome evolution includes structural rearrangement. J Bacteriol. 2017;199:199. https://doi.org/10.1128/JB.00806-16

15. Barkoff A-M, Mertsola J, Pierard D, Dalby T, Hoegh SV, Guillot S, et al. Pertactin-deficient Bordetella pertussis isolates: evidence of increased circulation in Europe, 1998 to 2015. Euro Surveill. 2019;24:1700832. https:/ / doi.org/ 10.2807/1560-7917.ES.2019.24.7.1700832

16. Sealey KL, Harris SR, Fry NK, Hurst LD, Gorringe AR, Parkhill J, et al. Genomic analysis of isolates from the United Kingdom 2012 pertussis outbreak reveals that vaccine antigen genes are unusually fast evolving. J Infect Dis. 2015;212:294-301. https:/ / doi.org/10.1093/infdis/jiu665

17. Vestrheim DF, Steinbakk M, Bjørnstad ML, Moghaddam A, Reinton N, Dahl ML, et al. Recovery of Bordetella pertussis from PCR-positive nasopharyngeal samples is dependent on bacterial load. J Clin Microbiol. 2012;50:4114-5. https://doi.org/10.1128/JCM.01553-12

18. Martini H, Rodeghiero C, VAN DEN Poel C, Vincent M, Pierard D, Huygen K. Pertussis diagnosis in Belgium: results of the National Reference Centre for Bordetella anno 2015. Epidemiol Infect. 2017;145:2366-73. https:/ / doi.org/ 10.1017/S0950268817001108

19. Lam C, Octavia S, Bahrame Z, Sintchenko V, Gilbert GL, Lan R. Selection and emergence of pertussis toxin promoter ptxP3 allele in the evolution of Bordetella pertussis. Infect Genet Evol. 2012;12:492-5. https://doi.org/10.1016/ j.meegid.2012.01.001

20. Bowden KE, Williams MM, Cassiday PK, Milton A, Pawloski L, Harrison M, et al. Molecular epidemiology of the pertussis epidemic in Washington State in 2012. J Clin Microbiol. 2014;52:3549-57. https:// doi.org/10.1128/ JCM.01189-14

21. van Gent $M$, Heuvelman $C J$, van der Heide HG, Hallander HO, Advani A, Guiso N, et al. Analysis of Bordetella pertussis clinical isolates circulating in European countries during the period 1998-2012. Eur J Clin Microbiol Infect Dis. 2015;34:821-30. https://doi.org/10.1007/ s10096-014-2297-2

22. Bart MJ, Harris SR, Advani A, Arakawa Y, Bottero D, Bouchez V, et al. Global population structure and evolution of Bordetella pertussis and their relationship with vaccination. MBio. 2014;5:e01074-14. https:// doi. org/10.1128/mBio.01074-14

23. Moriuchi T, Vichit O, Vutthikol Y, Hossain MS, Samnang C, Toda K, et al. Molecular epidemiology of Bordetella pertussis in Cambodia determined by direct genotyping of clinical specimens. Int J Infect Dis. 2017;62:56-8. https://doi.org/10.1016/j.ijid.2017.07.015

24. Miyaji Y, Otsuka N, Toyoizumi-Ajisaka H, Shibayama K, Kamachi K. Genetic analysis of Bordetella pertussis isolates from the 2008-2010 pertussis epidemic in Japan. PLoS One. 2013;8:e77165. https:// doi.org/10.1371/journal. pone. 0077165

25. Breakwell L, Kelso P, Finley C, Schoenfeld S, Goode B, Misegades LK, et al. Pertussis vaccine effectiveness in the setting of pertactin-deficient pertussis. Pediatrics. 2016;137:e20153973. https:/ / doi.org/10.1542/peds.2015-3973

26. Etskovitz H, Anastasio N, Green E, May M. Role of evolutionary selection scting on vaccine antigens in the re-emergence of Bordetella pertussis. Diseases. 2019;7:35. https://doi.org/10.3390/diseases7020035

27. Safarchi A, Octavia S, Nikbin VS, Lotfi MN, Zahraei SM, Tay CY, et al. Genomic epidemiology of Iranian Bordetella pertussis: 50 years after the implementation of whole cell vaccine. Emerg Microbes Infect. 2019;8:1416-27. https:/ / doi.org/10.1080/22221751.2019.1665479

28. Carriquiriborde F, Regidor V, Aispuro PM, Magali G, Bartel E, Bottero D, et al. Rare detection of Bordetella pertussis pertactin-deficient strains in Argentina. Emerg Infect Dis. 2019;25:2048-54. https:// doi.org/10.3201/ eid2511.190329

29. Safarchi A, Octavia S, Luu LD, Tay CY, Sintchenko V, Wood N, et al. Pertactin negative Bordetella pertussis demonstrates higher fitness under vaccine selection pressure in a mixed infection model. Vaccine. 2015;33:627781. https:/ / doi.org/10.1016/j.vaccine.2015.09.064

30. Carvalho CFA, Andrews N, Dabrera G, Ribeiro S, Stowe J, Ramsay M, et al. National Outbreak of Pertussis in England, 2011-2012: a case-control study comparing 3-component and 5-component acellular vaccines with whole-cell pertussis vaccines. Clin Infect Dis. 2020;70:200-7. https://doi.org/10.1093/cid/ciz199

31. van Twillert I, Bonačić Marinović AA, Kuipers B, van Gaans-van den Brink JAM, Sanders EAM, van Els CACM. Impact of age and vaccination history on long-term serological responses after symptomatic B. pertussis infection, a high dimensional data analysis. Sci Rep. 2017;7:40328. https:// doi.org/10.1038/srep40328

32. Olin P, Rasmussen F, Gustafsson L, Hallander HO, Heijbel H; Ad Hoc Group for the Study of Pertussis Vaccines. Randomised controlled trial of two-component, three-component, and five-component acellular pertussis vaccines compared with whole-cell pertussis vaccine. Lancet. 1997;350:1569-77. https:// doi.org/10.1016/ S0140-6736(97)06508-2

33. Zeddeman A, van Gent M, Heuvelman CJ, van der Heide HG, Bart MJ, Advani A, et al. Investigations into the emergence of pertactin-deficient Bordetella pertussis isolates in six European countries, 1996 to 2012. Euro Surveill. 2014;19:20881. https://doi.org/10.2807/ 1560-7917.ES2014.19.33.20881

34. Weigand MR, Williams MM, Peng Y, Kania D, Pawloski LC, Tondella ML; CDC Pertussis Working Group. Genomic survey of Bordetella pertussis diversity, United States, 2000-2013. Emerg Infect Dis. 2019;25:780-3. https://doi.org/10.3201/eid2504.180812 
35. Xu Z, Octavia S, Luu LDW, Payne M, Timms V, Tay CY, et al. Pertactin-negative and filamentous hemagglutininnegative Bordetella pertussis, Australia, 2013-2017. Emerg Infect Dis. 2019;25:1196-9. PubMed https://doi.org/10.3201/ eid2506.180240

36. Weigand MR, Peng Y, Cassiday PK, Loparev VN, Johnson T, Juieng P, et al. Complete genome sequences of Bordetella pertussis isolates with novel pertactin-deficient deletions. Genome Announc. 2017;5:e00973-17. https:/ / doi.org/10.1128/genomeA.00973-17

37. Tsang RS, Shuel M, Jamieson FB, Drews S, Hoang L, Horsman G, et al. Pertactin-negative Bordetella pertussis strains in Canada: characterization of a dozen isolates based on a survey of 224 samples collected in different parts of the country over the last 20 years. Int J Infect Dis. 2014;28:65-9. https:/ / doi.org/10.1016/j.ijid.2014.08.002
38. Lam C, Octavia S, Ricafort L, Sintchenko V, Gilbert GL, Wood N, et al. Rapid increase in pertactin-deficient Bordetella pertussis isolates, Australia. Emerg Infect Dis. 2014;20:626-33. https://doi.org/10.3201/eid2004.131478

39. Uelze L, Grützke J, Borowiak M, Hammerl JA, Juraschek K, Deneke C, et al. Typing methods based on whole genome sequencing data. One Health Outlook. 2020;2:3 https://doi.org/10.1186/s42522-020-0010-1

40. Jolley KA, Bray JE, Maiden MCJ. Open-access bacterial population genomics: BIGSdb software, the PubMLST.org website and their applications. Wellcome Open Res. 2018;3:124 https://doi.org/10.12688/wellcomeopenres.14826.1

Address for correspondence: Adriana Cabal Rosel, Austrian Agency for Health and Food Safety, Währingerstraße 25a, 1096 Vienna, Austria; email: adriana@cabalrosel.com

\section{Emerging Infectious Diseases Spotifght Topics}
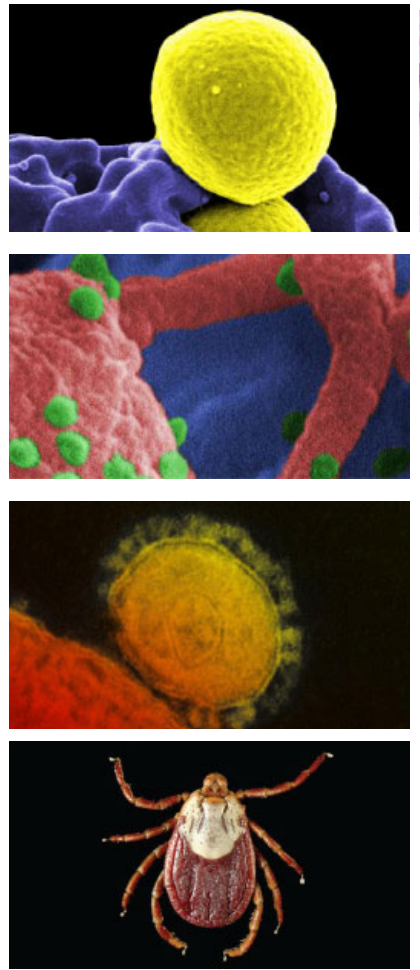

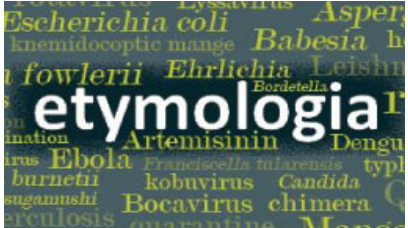

Antimicrobial resistance • Ebola Etymologia • Food safety • HIV-AIDS Influenza • Lyme disease • Malaria MERS • Pneumonia • Rabies • Ticks Tuberculosis • Coronavirus • Zika
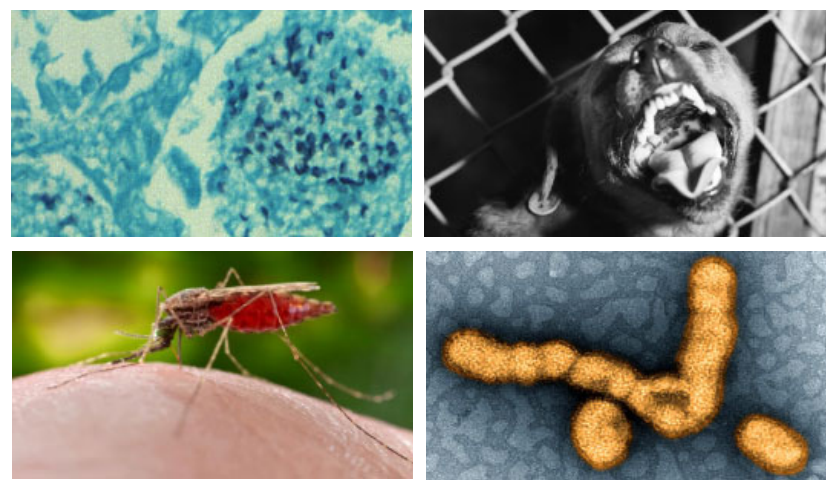
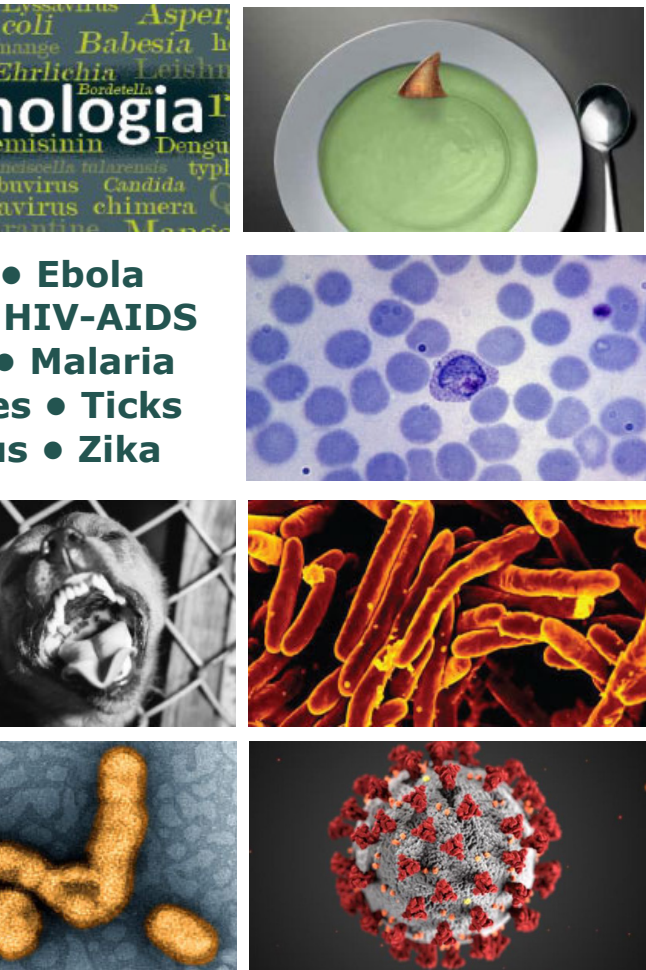

EID's spotlight topics highlight the latest articles and information on emerging infectious disease topics in our global community

https://wwwnc.cdc.gov/eid/page/spotlight-topics 